\title{
Parkinson's Disease and Systemic Inflammation
}

\author{
Carina C. Ferrari and Rodolfo Tarelli \\ Laboratorio de Terapias Regenerativas y Protectoras del Sistema Nervioso, Fundación Instituto Leloir, Patricias Argentinas 435, \\ C1405BWE Buenos Aires, Argentina
}

Correspondence should be addressed to Carina C. Ferrari, cferrari@leloir.org.ar

Received 15 October 2010; Accepted 7 January 2011

Academic Editor: Carlos Barcia

Copyright $\odot 2011$ C. C. Ferrari and R. Tarelli. This is an open access article distributed under the Creative Commons Attribution License, which permits unrestricted use, distribution, and reproduction in any medium, provided the original work is properly cited.

\begin{abstract}
Peripheral inflammation triggers exacerbation in the central brain's ongoing damage in several neurodegenerative diseases. Systemic inflammatory stimulus induce a general response known as sickness behaviour, indicating that a peripheral stimulus can induce the synthesis of cytokines in the brain. In Parkinson's disease (PD), inflammation was mainly associated with microglia activation that can underlie the neurodegeneration of neurons in the substantia nigra (SN). Peripheral inflammation can transform the "primed" microglia into an "active" state, which can trigger stronger responses dealing with neurodegenerative processes. Numerous evidences show that systemic inflammatory processes exacerbate ongoing neurodegeneration in PD patient and animal models. Anti-inflammatory treatment in PD patients exerts a neuroprotective effect. In the present paper, we analyse the effect of peripheral infections in the etiology and progression in PD patients and animal models, suggesting that these peripheral immune challenges can exacerbate the symptoms in the disease.
\end{abstract}

\section{Neurodegenerative Diseases and Systemic Inflammation}

Inflammation is a defensive reaction against harmful stimuli that can induce a defensive response in the body. In the central nervous system (CNS), the main innate immune defensive role is played by the immunocompetent resident cells, the microglia [1]. Neurodegenerative diseases present microglia activation as the main hallmark, which can change its morphology from quiescent and ramified (resting) towards a round ameboidal shape (activated) [2]. Resting microglia displays a low-level expression of membrane receptors, such as CD45, CD14, and CD11b [1]. Activated microglia exhibits upregulation of cell surface receptors and proinflammatory and anti-inflammatory cytokines, such as major histocompatibility complex (MHC) class II, CD40, CD80, CD86, CD11b (reviewed by [3]) demonstrating changes in their activity $[4,5]$ (Figure 1). Microglia can be activated by proinflammatory stimuli, but microglia activation does not always exert a proinflammatory reaction. Microglial activation in some neurodegenerative diseases was not accompanied by proinflammatory cytokine secretions
$[6,7]$. Depino et al., 2003 demonstrated that microglial cells induced an increase in IL- $1 \beta$ mRNA in the substantia nigra (SN) but no translation of this cytokine was observed in an animal model of PD. These observations prompted the idea of "primed microglia" to describe the atypical microglia state, which precedes a further neurotoxic microglial activation as a consequence of a secondary proinflammatory stimulus $[8,9]$. Microglia activation increases neurotoxicity and, therefore, contributes to neurodegeneration through the release of free radicals such as superoxide radicals, nitric Oxide (NO), inducible nitric oxide synthase (iNOS) [10-13], and proinflammatory, immunomodulatory and anti-inflammatory cytokines, such as IL- $1 \beta$, TNF- $\alpha$, IL6, IL-8, IL-12, IL-15, and IL-10 $[4,14,15]$. Central or peripheral inflammation can transform the "primed" state of microglia into an "active" state, which can trigger or induce stronger responses dealing with neurodegenerative processes. Therefore, inflammation was mainly associated with microglia activation that can underlie the neurodegeneration of dopaminergic neurons of the SN.

The CNS has been considered as immunologically privileged and protected by the blood brain barrier (BBB) which 


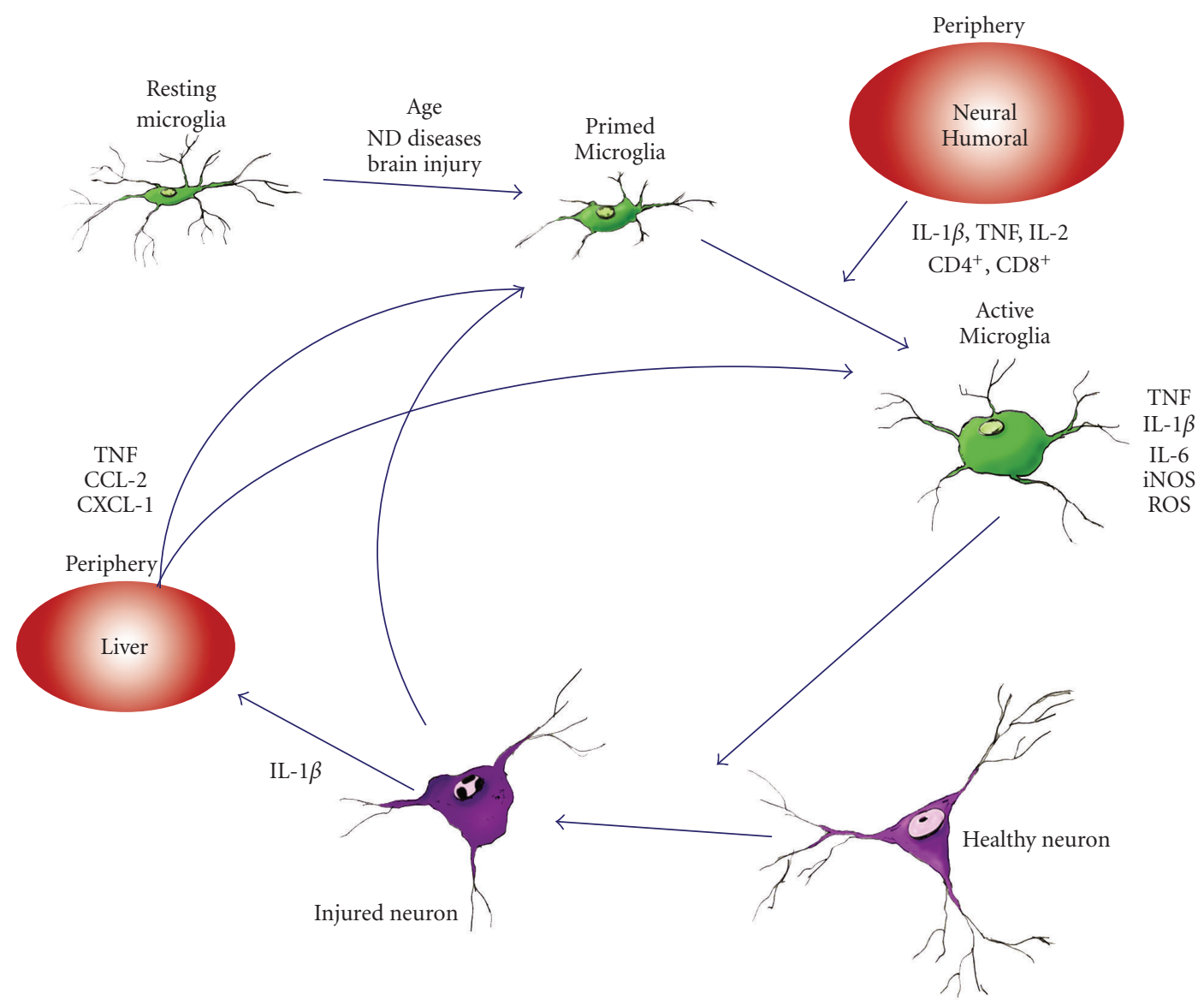

FIGURE 1: Schematic diagram showing the relationship between peripheral inflammation and neuronal loss in PD. Neurodegenerative diseases present microglial activation as the main hallmark, which can change its morphology from resting (ramified) towards an activated round shape (ameboidal). The intermediate stage, "primed microglia", describes the atypical microglial stage, which precedes a further neurotoxic microglial activation as a consequence of a secondary pro-inflammatory stimulus. This stimulus can come from the periphery, either through neural or humoral pathways. Activated microglia release pro-inflammatory cytokines which can act on neuronal integrity. In addition, acute brain injury induces early hepatic expression of chemokines, which in turn produce recruitment of leukocytes into the blood and subsequently brain and liver inflammation via a chemokines and cytokines web.

prevents entry of pathogens and immune cells into the parenchyma. However, this statement has changed in the last few years, because the communication between central CNS and periphery is more fluid than previously considered. In many neurological disorders, the immune system plays an important role in the progression of these diseases. Indeed, BBB breakdown and inflammation appear to play a major role in the pathology of numerous neurodegenerative diseases compromising the vascular unit and inducing leukocyte migration within the brain parenchyma (reviewed in [16]).

Systemic inflammatory stimuli circulate into the blood and can get into the brain inducing the synthesis of cytokines that, in turn, can induce a general inflammatory response including liver acute phase response and the components to induce sickness behaviour [17-21]. Proinflammatory stimulus would trigger the secretion of proinflammatory molecules in the diseased brain [6] (Figure 1).

Peripheral inflammation sparks off exacerbation in the central brain's ongoing damage in several neurodegenerative diseases, such as Alzheimer's disease (AD), multiple sclerosis, Parkinson's disease, prion disease, stroke, and Wallerian degeneration $[8,9,22-26]$. Indeed, Perry's group has studied the effect of peripheral inflammation on behavioural response, demonstrating a worsening of degenerative processes related to delirium in $\mathrm{AD}[19,27]$. In particular, PD patients and animal models with ongoing inflammatory neurodegeneration processes evidence exacerbation of the neurodegenerative process after a peripheral inflammatory stimulus [28-33]. Aging was also proposed to prime microglia cells [14]. MHC-II was increased in aged brains and i.p administration of LPS resulted in an increased inflammatory response in elderly patients $[15,34-36]$.

Previously, we described that the communication between the brain and the periphery as a one way road. However, the central-peripheral relationship is more complex, and the traffic becomes a two way road. Acute brain injury induces early hepatic expression of chemokines, which in turn produce movement of leukocytes into the blood and subsequently brain and liver inflammation $[9,37-40]$. The 
production of cytokines by the liver as a systemic response to CNS injury is a component of CNS response. The injection of IL-1 into the brain is associated with hepatic expression of CXCL1, which is responsible for neutrophil recruitment to the brain [40]. Hepatic TNF- $\alpha$ is also a component of the systemic response to IL- $1 \beta$ injured brain [39]. TNF- $\alpha$ was also found associated with IL- $1 \beta$ induced sickness behaviour, in addition, the inhibition of peripheral TNF- $\alpha$ can block some components of sickness behaviour induced by centrally injected IL-1 $\beta$ [41]. Peripheral TNF- $\alpha$ appears to be involved in microglial activation and the subsequent recruitment of monocytes into the brain in a model of peripheral liver inflammation resulting from bile duct ligation [42]. Therefore, the hepatic production of cytokines and chemokines may also be considered as a target to neutralize acute and chronic brain injury (Figure 1).

In summary, systemic inflammatory events could influence the aetiology and progression of many ongoing degenerative diseases. Despite previous evidences, the contribution of peripheral inflammation to the progression of neurodegenerative diseases is not fully understood. Analysis of the inflammatory components of the systemic response that influence ongoing damage in the brain should be carefully studied and considered as potential therapeutic targets.

\section{Routes of Systemic Inflammation}

The circulating cytokines and other inflammatory molecules that are produced by systemically induced insults can affect the brain through several routes, mainly humoral or neural pathways. The humoral route between the nervous and the immune systems has been related to sickness behaviour, characterized by fever, anorexia, and alteration in the behaviour. The humoral mechanisms are mostly related to the presence of the blood brain barrier (BBB). The BBB regulates the passage of substances from the blood to the brain (reviewed in [43]). This barrier can be seriously affected in brain injury. There are several ways of crossing the barrier: (1) the substances can enter through the areas in the CNS that lack BBB, like the circumventricular organs, (2) some molecules may cross the BBB using specific transporters (e.g., cytokines, amines), (3) BBB permeability may be increased as a consequence of the stimuli per se, (4) endothelial cells can be activated by the peripheral stimuli, inducing the synthesis of molecules within the CNS [4446], and (5) the choroidplexus transiently alters its gene expression profile as a response to peripheral LPS stimuli [47]. Systemic injection of LPS can cause BBB damage and allow the entrance of granulocytes from the periphery to the brain [48]. Indeed, BBB breakdown was described in PD patients and animal models [49-53]. The disruption of the $\mathrm{BBB}$ allows the extravasation of proinflammatory cytokines and immune cells which can activate microglia in the SN and, therefore, induce neurodegeneration.

The second route of neuroimmune communication, known as the neural pathway, is related to the transmission of peripheral inflammatory signals through the autonomic nervous system. The most important afferent responsible for the neural transmission of peripheral signals is the vagus nerve. Neural pathways are stimulated by peripheral signals that rapidly increase the levels of brain cytokines $[2,54,55]$. Subdiaphragmatic lesion of the vagus nerve and vagotomy attenuate brain cytokine production and behavioural effects after a systemic challenge [56-60]. IL$1 \beta$ receptors are present on vagal ganglia close to liver and lymphatic nodes [61]. Inflammatory processes in the periphery are conducted to the brain via the vagal afferents and, as a response, the vagal efferents act on the systemic inflammatory events through acetylcholinesterase secretions $[25,54,62]$. In addition, Kamer et al., 2008, suggested that the neural pathway is also involved in the transmission of inflammatory signals from the oral cavity in periodontal diseases, and this mechanism would be related to worsening of AD symptoms [62].

A third potential pathway was recently proposed using a model of inflammatory liver injury [42]. These authors suggested the existence of cellular messengers, activated monocytes, which were recruited into the brain. These activated monocytes secreted messenger molecules, such as TNF- $\alpha$ and MCP-1 (which has been classically defined as humoral) within the brain during systemic inflammatory diseases $[42,63]$.

\section{Parkinson's Disease and Systemic Inflammation: Evidence from the Clinic}

Peripheral immunological challenges and chronic inflammatory diseases influence the pathogenesis and progression of PD. The communication between the immune and the nervous system is very fluid, cytokines being the main mediators of inflammation in both brain and periphery. There is evidence that suggests a link between peripheral inflammation and PD. The influenza pandemic during the second world war was associated with an increase in $\mathrm{PD}$ in the population [64]. In addition, people infected with Japanese encephalitis virus and $\mathrm{H} 5 \mathrm{~N} 1$ influenza virus presented a higher risk for developing PD $[65,66]$.

Activated microglial cells and proinflammatory cytokines, including IL- $1 \beta$, TNF- $\alpha$, and IL- 6 , have been described in $\mathrm{SN}$ of postmortem tissue $[67,68]$, as reviewed in [69]. In vivo studies have also demonstrated that the serum and cerebrospinal fluid of PD patients have higher levels of IL- $1 \beta$, TNF- $\alpha$, and IL- 2 and also $\mathrm{CD}^{+}$and $\mathrm{CD}^{+}$T lymphocytes, indicating peripheral activation of lymphocytes [70-74]. The relationship between inflammation and $\mathrm{PD}$ has been demonstrated by several authors [3, 69, 75-79]. Subject carriers of IL- $1 \beta-511$ homozygous variant genotype show a 2 -fold increased risk of $\mathrm{PD}$, which induced an increment of susceptibility of dopaminergic neurons to toxicity [80]. On the other hand, increased peripheral cytokine production influences PD progression. PD patients showed elevated serum levels of TNF- $\alpha$ and TNF- $\alpha$ receptor 1 compared to control subjects, which can contribute to PD pathogenesis $[72,81,82]$. Also, elevated plasma concentration of IL-6 correlates with increased risk of PD [52].

Although late onset sporadic PD was recently associated with genetic variation in the HLA DR region, stressing the importance of the immune component in this disease, 
[83] PD can also be triggered by diseases that induce systemic infections. Indeed, PD patients often suffer infectious diseases, and the main causes of death are pneumonia and respiratory infections [30-33]. It was described that gastrointestinal infections could contribute to a worsening of $\mathrm{PD}[84,85]$. However, peripheral immune response in $\mathrm{PD}$ patients has shown contradictory results, because some authors have found unaffected levels of cytokines and immune parameters in PD patients [73, 86]. Several studies support a role for the adaptative immune system in $\mathrm{PD}$ etiology and progression. The presence of cytotoxic $\mathrm{T}$ lymphocyte $\left(\mathrm{CD}^{+}\right.$and $\mathrm{CD}^{+}$) has been described to infiltrate the SN of patients and animal PD models [8789]. The influx of these peripheral cells into the brain parenchyma could indicate a BBB dysfunction in $\mathrm{PD}$ patients $[49,69]$. Indeed, the adaptative immune system might modulate microglia activation in $\mathrm{PD}$ pathogenesis [90].

Anti-inflammatory therapies were used in PD patients to decrease the effect of inflammatory reactions. The NSAIDs had an anti-inflammatory and neuroprotective effect in PD. Chronic users of nonsteroidal anti-inflammatory drugs (NSAIDs), such as ibuprofen, a COX-1 and COX-2 inhibitor, exhibited diminished PD incidence $[91,92]$. Minocycline, a derivate of tetracycline that crosses the $\mathrm{BBB}$, also improves neuronal survival in PD. This molecule was described as an inhibitor of microglial activation, proliferation, and release of proinflammatory cytokines [93-95]. It should be taken into consideration that inflammation has a dual action in neurodegeneration, also inducing molecules useful in promoting repair and regeneration of damaged tissue. Therefore, considering the beneficial effects of some of the inflammatory components, restriction of the inflammatory response is not always the best choice. Better alternative therapies should be considered in order to really make progress in this field (reviewed in [96]). These authors propose a multidisciplinary research aimed at protein clearance and immunoprotection induced by $\mathrm{T}$ cell regulators.

The studies related to the inflammatory processes in PD should be carefully evaluated in order to develop more suitable tools that will allow us to diminish neurodegeneration and improve the quality of life in PD patients.

\section{Parkinson's Disease and Systemic Inflammation: Experimental Evidences}

The link between peripheral infection and PD neurodegeneration in both patient and animal models was demonstrated in several studies. LPS causes a systemic inflammatory reaction known as sickness behaviour characterized by fever, anorexia, weight loss, and reduction of activity [97]. A similar response can be obtained with i.p injection of proinflammatory cytokines, such as IL- $1 \beta$ and IL- 6 (reviewed in [58]). The resident microglia in the brain responds to these stimuli and generates sickness behaviour. Taking this into consideration, ongoing inflammatory degenerative processes can be accelerated by systemic inflammation. Peripheral inflammatory states, such as infection and injury, can exacerbate neuronal death stimulating "primed microglial cells" towards a more aggressive state.
PD animal models support the previous hypothesis. Pregnant rats exposed to intraperitoneal (i.p.) injection of LPS resulted in a decreased number of dopaminergic neurons in the pups when compared to nonexposed controls [98]. In concordance with Carvey's results, rat foetuses exposed to LPS are more susceptible to 6-OHDA in adulthood [99, 100]. In adult animals, there is also data that strongly suggests the role of peripheral inflammation in the ongoing PD model. Animals with central dopaminergic hypoactivity are associated with an increased peripheral inflammatory response after bacterial LPS injection [101]. Gastrointestinal dysfunctions are related to peripheral inflammation; indeed, ulcerative colitis correlates with increased levels of TNF- $\alpha$, IL- $1 \beta$, IL-6, and acute phase proteins in rat serum [102]. Peripheral inflammation induced by ulcerative colitis worsened the effects induced by intranigral LPS, such as loss of dopaminergic neurons, microglial activation, and alteration in BBB permeability [102]. All previous data indicate that the relationship between the peripheral immune system and the central dopaminergic system is very close.

Proinflammatory cytokines, including IL- $1 \beta$ and TNF, have been described as involved in promoting neurodegeneration. These cytokines induced the synthesis of chemokines, producing in turn the recruitment of neutrophils and monocytes from the blood stream. IL- $1 \beta$ alone might induce the cellular recruitment to the brain parenchyma [103-106]. The effects of IL- $1 \beta$ in the progression of neurodegenerative disease have been studied by several groups [9, 23, 28, 29, 3739, 104, 105, 107]. Systemic challenge with LPS induces CNS IL- $1 \beta$ synthesis and sickness behaviour in animals with ongoing central inflammation [19]. However, systemic inflammation actively inhibits recruitment of leukocytes in the CNS, when LPS is injected 2 hours before the intracerebroventricular injection of IL-1 $\beta$ [108]. Systemic inflammation generated by IL- $1 \beta$ induces $\mathrm{BBB}$ disruption and increased brain damage in a model of stroke $[9,23]$. In addition, chronic systemic expression of IL- $1 \beta$ was able to exacerbate neuronal demise and microglial activation in the SN of both 6OHDA and an inflammatory PD model $[28,29]$, increasing the clinical impact of these findings [29]. Exacerbation of neurodegeneration in 6OHDA model was accompanied by an increase in activated microglia in the animals that received IL-1 $\beta$ as peripheral stimulus [28]. The same group has demonstrated that a peripheral systemic stimulus causes exacerbation of the behavioral symptoms and neuronal loss in an inflammatory PD model based on the chronic IL- $1 \beta$ expression in the striatum. These events were accompanied by massive activation of microglia with the concomitant expression of MHC-II [29]. Therefore, the increment in the neurodegenerative process can be correlated with an increase in MHC-II expression induced by a peripheral stimulus [29].

Peripheral induction of TNF- $\alpha$ activates brain microglia that, in turn, produces proinflammatory factors and, as a consequence, induces dopaminergic neuronal loss in the SN [109]. Indeed, dominant negative TNF- $\alpha$ inhibitor displayed neuroprotective properties in both 6OHDA and chronic LPS rat model [110]. 
However, on the contrary, some inflammatory components of the adaptative immune response were described as immunomodulators of the neurodegenerative process. $\mathrm{CD}^{+} \mathrm{CD}^{2} 5^{+}$regulatory $\mathrm{T}$ cells (Tregs) suppress neuroinflammation, attenuate microglia response, and induce nigrostriatal protection in a MPTP model [111, 112]. These cells exert their activity by upregulation of brain-derived neurotrophic factor (BDNF), glial-derived neurotrophic factor (GDNF), IL-10 and transforming growth factor (TGF- $\beta$ ), and downregulating proinflammatory cytokines and ROS production (reviewed in $[111,113]$ ). These data suggest that Tregs exert their action modulating the immune response, possibly via the interaction between the peripheral and the CNS immune system (Reviewed in $[89,96])$. Immunotherapy should be directed towards a Th2 Treg response in order to downregulate the Th1 response [112]. The interaction of Tregs with cells or molecules may modulate the adaptative immune response. Therefore, alterations of the immune system as a consequence of peripheral inflammation could change the immunological properties of Tregs, inducing a phenotype not suitable for the beneficial Treg activation. Further studies should be undertaken before Tregs immunization treatment is to be considered for PD patients with ongoing peripheral infections.

The use of anti-inflammatory drugs has been extensively studied in PD animal models (reviewed in [78]). The role of COX has been studied in an MPTP model demonstrating that indomethacin protects dopaminergic neurons in SN [114]. Treatment with rofecoxib and celecoxib also induces a protective effect in the SN neurons [115-117]. In addition, the use of dexamethasone was demonstrated to reduce the neuronal loss in a 6-OHDA plus LPS exacerbation and, in an inflammatory model of PD, exacerbated with IL- $1 \beta$ as a peripheral inflammatory stimulus $[28,29]$.

The interaction between brain inflammation and systemic inflammation may be responsible for the progression of neurodegenerative disease. Studying the relationship between CNS and periphery could help find targets for therapeutic treatments.

\section{Conclusion}

Central or systemic inflammatory insults should be considered as risk factors in the PD aetiology and progression. The clear knowledge of mechanisms implicated in immune/nervous communication and the mechanisms involved in microglia activation and their switch to an aggressive phenotype could help improve the therapeutic tools leading to better patient quality of life, reducing the exacerbation of PD symptoms, and delaying the progression of the disease.

\section{References}

[1] G. W. Kreutzberg, "Microglia: a sensor for pathological events in the CNS," Trends in Neurosciences, vol. 19, no. 8, pp. 312-318, 1996.
[2] V. H. Perry, J. A. R. Nicoll, and C. Holmes, "Microglia in neurodegenerative disease," Nature Reviews Neurology, vol. 6, no. 4, pp. 193-201, 2010.

[3] P. S. Whitton, "Inflammation as a causative factor in the aetiology of Parkinson's disease," British Journal of Pharmacology, vol. 150, no. 8, pp. 963-976, 2007.

[4] S. U. Kim and J. de Vellis, "Microglia in health and disease," Journal of Neuroscience Research, vol. 81, no. 3, pp. 302-313, 2005.

[5] R. M. Ransohoff and V. H. Perry, "Microglial physiology: unique stimuli, specialized responses," Annual Review of Immunology, vol. 27, pp. 119-145, 2009.

[6] V. H. Perry, C. Cunningham, and D. Boche, "Atypical inflammation in the central nervous system in prion disease," Current Opinion in Neurology, vol. 15, no. 3, pp. 349-354, 2002.

[7] A. M. Depino, C. Earl, E. Kaczmarczyk et al., "Microglial activation with atypical proinflammatory cytokine expression in a rat model of Parkinson's disease," European Journal of Neuroscience, vol. 18, no. 10, pp. 2731-2742, 2003.

[8] C. Cunningham, D. C. Wilcockson, S. Campion, K. Lunnon, and V. H. Perry, "Central and systemic endotoxin challenges exacerbate the local inflammatory response and increase neuronal death during chronic neurodegeneration," Journal of Neuroscience, vol. 25, no. 40, pp. 9275-9284, 2005.

[9] B. W. McColl, N. J. Rothwell, and S. M. Allan, "Systemic inflammatory stimulus potentiates the acute phase and CXC chemokine responses to experimental stroke and exacerbates brain damage via interleukin-1- and neutrophil-dependent mechanisms," Journal of Neuroscience, vol. 27, no. 16, pp. 4403-4412, 2007.

[10] T. Arimoto and G. Bing, "Up-regulation of inducible nitric oxide synthase in the substantia nigra by lipopolysaccharide causes microglial activation and neurodegeneration," Neurobiology of Disease, vol. 12, no. 1, pp. 35-45, 2003.

[11] M. M. Iravani, K. Kashefi, P. Mander, S. Rose, and P. Jenner, "Involvement of inducible nitric oxide synthase in inflammation-induced dopaminergic neurodegeneration," Neuroscience, vol. 110, no. 1, pp. 49-58, 2002.

[12] A. Czlonkowska, I. Kurkowska-Jastrzebska, A. Czlonkowski, D. Peter, and G. B. Stefano, "Immune processes in the pathogenesis of Parkinson's disease-a potential role for microglia and nitric oxide," Medical Science Monitor, vol. 8, no. 8, pp. RA165-RA177, 2002.

[13] L. Minghetti, E. Polazzi, A. Nicolini, A. Greco, and G. Levi, "Possible role of microglial prostanoids and free radicals in neuroprotection and neurodegeneration," Advances in Experimental Medicine and Biology, vol. 468, pp. 109-119, 1999.

[14] R. N. Dilger and R. W. Johnson, "Aging, microglial cell priming, and the discordant central inflammatory response to signals from the peripheral immune system," Journal of Leukocyte Biology, vol. 84, no. 4, pp. 932-939, 2008.

[15] C. J. Henry, Y. Huang, A. M. Wynne, and J. P. Godbout, "Peripheral lipopolysaccharide (LPS) challenge promotes microglial hyperactivity in aged mice that is associated with exaggerated induction of both pro-inflammatory IL-1 $\beta$ and anti-inflammatory IL-10 cytokines," Brain, Behavior, and Immunity, vol. 23, no. 3, pp. 309-317, 2009.

[16] H. B. Stolp and K. M. Dziegielewska, "Review: role of developmental inflammation and blood-brain barrier dysfunction in neurodevelopmental and neurodegenerative diseases," Neuropathology and Applied Neurobiology, vol. 35, no. 2, pp. 132-146, 2009. 
[17] H. O. Besedovsky and A. del Rey, "Immune-neuro-endocrine interactions: facts and hypotheses," Endocrine Reviews, vol. 17, no. 1, pp. 64-102, 1996.

[18] F. Pitossi, A. del Rey, A. Kabiersch, and H. Besedovsky, "Induction of cytokine transcripts in the central nervous system and pituitary following peripheral administration of endotoxin to mice," Journal of Neuroscience Research, vol. 48, no. 4, pp. 287-298, 1997.

[19] M. I. Combrinck, V. H. Perry, and C. Cunningham, "Peripheral infection evokes exaggerated sickness behaviour in preclinical murine prion disease," Neuroscience, vol. 112, no. 1, pp. 7-11, 2002.

[20] V. H. Perry, "The influence of systemic inflammation on inflammation in the brain: implications for chronic neurodegenerative disease," Brain, Behavior, and Immunity, vol. 18, no. 5, pp. 407-413, 2004.

[21] D. Londono and D. Cadavid, "Bacterial lipoproteins can disseminate from the periphery to inflame the brain," American Journal of Pathology, vol. 176, no. 6, pp. 2848-2857, 2010.

[22] C. Cunningham, D. C. Wilcockson, D. Boche, and V. H. Perry, "Comparison of inflammatory and acute-phase responses in the brain and peripheral organs of the ME7 model of prion disease," Journal of Virology, vol. 79, no. 8, pp. 5174-5184, 2005.

[23] B. W. McColl, N. J. Rothwell, and S. M. Allan, "Systemic inflammation alters the kinetics of cerebrovascular tight junction disruption after experimental stroke in mice," Journal of Neuroscience, vol. 28, no. 38, pp. 9451-9462, 2008.

[24] S. J. Spencer, A. Mouihate, and Q. J. Pittman, "Peripheral inflammation exacerbates damage after global ischemia independently of temperature and acute brain inflammation," Stroke, vol. 38, no. 5, pp. 1570-1577, 2007.

[25] V. H. Perry, C. Cunningham, and C. Holmes, "Systemic infections and inflammation affect chronic neurodegeneration," Nature Reviews Immunology, vol. 7, no. 2, pp. 161-167, 2007.

[26] K. Palin, C. Cunningham, P. Forse, V. H. Perry, and N. Platt, "Systemic inflammation switches the inflammatory cytokine profile in CNS Wallerian degeneration," Neurobiology of Disease, vol. 30, no. 1, pp. 19-29, 2008.

[27] C. Cunningham, S. Campion, J. Teeling, L. Felton, and V. H. Perry, "The sickness behaviour and CNS inflammatory mediator profile induced by systemic challenge of mice with synthetic double-stranded RNA (poly I:C)," Brain, Behavior, and Immunity, vol. 21, no. 4, pp. 490-502, 2007.

[28] M. C. P. Godoy, R. Tarelli, C. C. Ferrari, M. I. Sarchi, and F. J. Pitossi, "Central and systemic IL-1 exacerbates neurodegeneration and motor symptoms in a model of Parkinson's disease," Brain, vol. 131, no. 7, pp. 1880-1894, 2008.

[29] M. C. Pott Godoy, C. C. Ferrari, and F. J. Pitossi, "Nigral neurodegeneration triggered by striatal AdIL-1 administration can be exacerbated by systemic IL-1 expression," Journal of Neuroimmunology, vol. 222, no. 1-2, pp. 29-39, 2010.

[30] Y. Hasegawa, T. Inagaki, M. Sawada, and A. Suzumura, "Impaired cytokine production by peripheral blood mononuclear cells and monocytes/macrophages in Parkinson's disease," Acta Neurologica Scandinavica, vol. 101, no. 3, pp. 159-164, 2000.

[31] M. K. Beyer, K. Herlofson, D. Arsland, and J. P. Larsen, "Causes of death in a community-based study of Parkinson's disease," Acta Neurologica Scandinavica, vol. 103, pp. 7-11, 2001.
[32] M. D’Amelio, P. Ragonese, L. Morgante et al., "Long-term survival of Parkinson's disease: a population-based study," Journal of Neurology, vol. 253, no. 1, pp. 33-37, 2006.

[33] H. Arai, T. Furuya, Y. Mizuno, and H. Mochizuki, "Inflammation and infection in Parkinson's disease," Histology and Histopathology, vol. 21, no. 4-6, pp. 673-678, 2006.

[34] J. P. Godbout, J. Chen, J. Abraham et al., "Exaggerated neuroinflammation and sickness behavior in aged mice following activation of the peripheral innate immune system," FASEB Journal, vol. 19, no. 10, pp. 1329-1331, 2005.

[35] J. Chen, J. B. Buchanan, N. L. Sparkman, J. P. Godbout, G. G. Freund, and R. W. Johnson, "Neuroinflammation and disruption in working memory in aged mice after acute stimulation of the peripheral innate immune system," Brain, Behavior, and Immunity, vol. 22, no. 3, pp. 301-311, 2008.

[36] M. Sawada, H. Sawada, and T. Nagatsu, "Effects of aging on neuroprotective and neurotoxic properties of microglia in neurodegenerative diseases," Neurodegenerative Diseases, vol. 5, no. 3-4, pp. 254-256, 2008.

[37] D. C. Wilcockson, S. J. Campbell, D. C. Anthony, and V. H. Perry, "The systemic and local acute phase response following acute brain injury," Journal of Cerebral Blood Flow and Metabolism, vol. 22, no. 3, pp. 318-326, 2002.

[38] S. J. Campbell, P. M. Hughes, J. P. Iredale et al., "CINC-1 is an acute-phase protein induced by focal brain injury causing leukocyte mobilization and liver injury," FASEB Journal, vol. 17, no. 9, pp. 1168-1170, 2003.

[39] S. J. Campbell, R. M. J. Deacon, Y. Jiang, C. Ferrari, F. J. Pitossi, and D. C. Anthony, "Overexpression of IL-1 $\beta$ by adenoviral-mediated gene transfer in the rat brain causes a prolonged hepatic chemokine response, axonal injury and the suppression of spontaneous behaviour," Neurobiology of Disease, vol. 27, no. 2, pp. 151-163, 2007.

[40] S. J. Campbell, V. H. Perry, F. J. Pitossi et al., "Central nervous system injury triggers Hepatic CC and CXC chemokine expression that is associated with leukocyte mobilization and recruitment to both the central nervous system and the liver," American Journal of Pathology, vol. 166, no. 5, pp. 1487-1497, 2005.

[41] Y. Jiang, R. Deacon, D. C. Anthony, and S. J. Campbell, "Inhibition of peripheral TNF can block the malaise associated with CNS inflammatory diseases," Neurobiology of Disease, vol. 32, no. 1, pp. 125-132, 2008.

[42] C. D’Mello, T. Le, and M. G. Swain, “Cerebral microglia recruit monocytes into the brain in response to tumor necrosis factora signaling during peripheral organ inflammation," Journal of Neuroscience, vol. 29, no. 7, pp. 2089-2102, 2009.

[43] B. V. Zlokovic, "The blood-brain barrier in health and chronic neurodegenerative disorders," Neuron, vol. 57, no. 2, pp. 178-201, 2008.

[44] R. Dantzer, "Cytokine-induced sickness behaviour: a neuroimmune response to activation of innate immunity," European Journal of Pharmacology, vol. 500, no. 1-3, pp. 399$411,2004$.

[45] R. Dantzer and K. W. Kelley, "Twenty years of research on cytokine-induced sickness behavior," Brain, Behavior, and Immunity, vol. 21, no. 2, pp. 153-160, 2007.

[46] L. E. Goehler, A. Erisir, and R. P. A. Gaykema, "Neuralimmune interface in the rat area postrema," Neuroscience, vol. 140, no. 4, pp. 1415-1434, 2006.

[47] F. Marques, J. C. Sousa, G. Coppola et al., "The choroid plexus response to a repeated peripheral inflammatory stimulus," BMC Neuroscience, vol. 10, article 135, 2009. 
[48] M. Bohatschek, A. Werner, and G. Raivich, "Systemic LPS injection leads to granulocyte influx into normal and injured brain: effects of ICAM-1 deficiency," Experimental Neurology, vol. 172, no. 1, pp. 137-152, 2001.

[49] R. Kortekaas, K. L. Leenders, J. C. H. van Oostrom et al., "Blood-brain barrier dysfunction in Parkinsonian midbrain in vivo," Annals of Neurology, vol. 57, no. 2, pp. 176-179, 2005.

[50] P. M. Carvey, C. H. Zhao, B. Hendey et al., "6hydroxydopamine-induced alterations in blood-brain barrier permeability," European Journal of Neuroscience, vol. 22, no. 5, pp. 1158-1168, 2005.

[51] C. Barcia, V. Bautista, A. Sanchez-Bahillo et al., "Changes in vascularization in substantia nigra pars compacta of monkeys rendered parkinsonian," Journal of Neural Transmission, vol. 112, no. 9, pp. 1237-1248, 2005.

[52] H. Chen, E. J. O’Reilly, M. A. Schwarzschild, and A. Ascherio, "Peripheral inflammatory biomarkers and risk of Parkinson's disease," American Journal of Epidemiology, vol. 167, no. 1, pp. 90-95, 2008.

[53] E. Yan, M. Castillo-Melendez, T. Nicholls, J. Hirst, and D. Walker, "Cerebrovascular responses in the fetal sheep brain to low-dose endotoxin," Pediatric Research, vol. 55, no. 5, pp. 855-863, 2004.

[54] K. J. Tracey, “The inflammatory reflex," Nature, vol. 420, no. 6917, pp. 853-859, 2002.

[55] V. H. Perry, T. A. Newman, and C. Cunningham, "The impact of systemic infection on the progression of neurodegenerative disease," Nature Reviews Neuroscience, vol. 4, no. 2, pp. 103-112, 2003.

[56] R. M. Bluthe, B. Michaud, K. W. Kelley, and R. Dantzer, "Vagotomy blocks behavioural effects of interleukin-1 injected via the intraperitoneal route but not via other systemic routes," NeuroReport, vol. 7, no. 15-17, pp. 28232827, 1996.

[57] R. M. Bluthe, B. Michaud, K. W. Kelley, and R. Dantzer, "Vagotomy attenuates behavioural effects of interleukin-1 injected peripherally but not centrally," NeuroReport, vol. 7, no. 9, pp. 1485-1488, 1996.

[58] R. Dantzer, R. M. Bluthe, S. Laye, J. L. Bret-Dibat, P. Parnet, and K. W. Kelley, "Cytokines and sickness behavior," Annals of the New York Academy of Sciences, vol. 840, pp. 586-590, 1998.

[59] R. M. Bluthe, V. Walter, P. Parnet et al., "Lipopolysaccharide induces sickness behaviour in rats by a vagal mediated mechanism," Comptes Rendus de l'Academie des Sciences, vol. 317, no. 6, pp. 499-503, 1994.

[60] M. K. Hansen, P. Taishi, Z. Chen, and J. M. Krueger, "Vagotomy blocks the induction of interleukin-1 $\beta$ (IL-1 $\beta$ ) mRNA in the brain of rats in response to systemic IL-1 $\beta$," Journal of Neuroscience, vol. 18, no. 6, pp. 2247-2253, 1998.

[61] L. E. Goehler, R. P. A. Gaykema, K. T. Nguyen et al., "Interleukin- $1 \beta$ in immune cells of the abdominal vagus nerve: a link between the immune and nervous systems?" Journal of Neuroscience, vol. 19, no. 7, pp. 2799-2806, 1999.

[62] A. R. Kamer, R. G. Craig, A. P. Dasanayake, M. Brys, L. Glodzik-Sobanska, and M. J. de Leon, "Inflammation and Alzheimer's disease: possible role of periodontal diseases," Alzheimer's and Dementia, vol. 4, no. 4, pp. 242-250, 2008.

[63] W. Rostene, P. Kitabgi, and S. M. Parsadaniantz, "Chemokines: a new class of neuromodulator?" Nature Reviews Neuroscience, vol. 8, no. 11, pp. 895-904, 2007.
[64] R. C. Dale, A. J. Church, R. A. H. Surtees et al., "Encephalitis lethargica syndrome: 20 new cases and evidence of basal ganglia autoimmunity," Brain, vol. 127, no. 1, pp. 21-33, 2004.

[65] H. Shoji, M. Watanabe, S. Itoh, H. Kuwahara, and F. Hattori, "Japanese encephalitis and parkinsonism," Journal of Neurology, vol. 240, no. 1, pp. 59-60, 1993.

[66] H. Jang, D. Boltz, K. Sturm-Ramirez et al., "Highly pathogenic $\mathrm{H} 5 \mathrm{~N} 1$ influenza virus can enter the central nervous system and induce neuroinflammation and neurodegeneration," Proceedings of the National Academy of Sciences of the United States of America, vol. 106, no. 33, pp. 14063-14068, 2009.

[67] M. Mogi, M. Harada, T. Kondob et al., "Interleukin-1 $\beta$, interleukin-6, epidermal growth factor and transforming growth factor- $\alpha$ are elevated in the brain from parkinsonian patients," Neuroscience Letters, vol. 180, no. 2, pp. 147-150, 1994.

[68] M. Mogi, M. Harada, H. Narabayashi, H. Inagaki, M. Minami, and T. Nagatsu, "Interleukin (IL)-1 $\beta$, IL-2, IL-4, IL- 6 and transforming growth factor- $\alpha$ levels are elevated in ventricular cerebrospinal fluid in juvenile parkinsonism and Parkinson's disease," Neuroscience Letters, vol. 211, no. 1, pp. 13-16, 1996.

[69] E. C. Hirsch and S. Hunot, "Neuroinflammation in Parkinson's disease: a target for neuroprotection?" Lancet Neurology, vol. 8, no. 4, pp. 382-397, 2009.

[70] R. J. Dobbs, A. Charlett, A. G. Purkiss, S. M. Dobbs, C. Weller, and D. W. Peterson, "Association of circulating TNF$\alpha$ and IL-6 with ageing and parkinsonism," Acta Neurologica Scandinavica, vol. 100, no. 1, pp. 34-41, 1999.

[71] D. Blum-Degena, T. Muller, W. Kuhn, M. Gerlach, H. Przuntek, and P. Riederer, "Interleukin- $1 \beta$ and interleukin-6 are elevated in the cerebrospinal fluid of Alzheimer's and de novo Parkinson's disease patients," Neuroscience Letters, vol. 202, no. 1-2, pp. 17-20, 1995.

[72] M. Reale, C. Iarlori, A. Thomas et al., "Peripheral cytokines profile in Parkinson's disease," Brain, Behavior, and Immunity, vol. 23, no. 1, pp. 55-63, 2009.

[73] K. Hisanaga, M. Asagi, Y. Itoyama, and Y. Iwasaki, "Increase in peripheral CD4 bright+ CD8 dull+T cells in Parkinson disease," Archives of Neurology, vol. 58, no. 10, pp. 1580-1583, 2001.

[74] J. Bas, M. Calopa, M. Mestre et al., "Lymphocyte populations in Parkinson's disease and in rat models of parkinsonism," Journal of Neuroimmunology, vol. 113, no. 1, pp. 146-152, 2001.

[75] M. G. Tansey, M. K. McCoy, and T. C. Frank-Cannon, "Neuroinflammatory mechanisms in Parkinson's disease: potential environmental triggers, pathways, and targets for early therapeutic intervention," Experimental Neurology, vol. 208, no. 1, pp. 1-25, 2007.

[76] T. C. Frank-Cannon, L. T. Alto, F. E. McAlpine, and M. G. Tansey, "Does neuroinflammation fan the flame in neurodegenerative diseases?" Molecular Neurodegeneration, vol. 4, no. 1, article 47, 2009.

[77] Y. C. Chung, H. W. Ko, E. Bok et al., "The role of neuroinflammation on the pathogenesis of Parkinson's disease," $B M B$ Reports, vol. 43, no. 4, pp. 225-232, 2010.

[78] E. Esposito, V. Di Matteo, A. Benigno, M. Pierucci, G. Crescimanno, and G. Di Giovanni, "Non-steroidal antiinflammatory drugs in Parkinson's disease," Experimental Neurology, vol. 205, no. 2, pp. 295-312, 2007. 
[79] J. B. Koprich, C. Reske-Nielsen, P. Mithal, and O. Isacson, "Neuroinflammation mediated by IL- $1 \beta$ increases susceptibility of dopamine neurons to degeneration in an animal model of Parkinson's disease," Journal of Neuroinflammation, vol. 5, article 8, 2008.

[80] A. D. Wahner, J. S. Sinsheimer, J. M. Bronstein, and B. Ritz, "Inflammatory cytokine gene polymorphisms and increased risk of Parkinson disease," Archives of Neurology, vol. 64, no. 6, pp. 836-840, 2007.

[81] P. Scalzo, A. Kummer, F. Cardoso, and A. L. Teixeira, "Increased serum levels of soluble tumor necrosis factor- $\alpha$ receptor-1 in patients with Parkinson's disease," Journal of Neuroimmunology, vol. 216, no. 1-2, pp. 122-125, 2009.

[82] M. Dufek, M. Hamanova, J. Lokaj et al., "Serum inflammatory biomarkers in Parkinson's disease," Parkinsonism and Related Disorders, vol. 15, no. 4, pp. 318-320, 2009.

[83] T. H. Hamza, C. P. Zabetian, A. Tenesa et al., "Common genetic variation in the HLA region is associated with lateonset sporadic Parkinson's disease," Nature Genetics, vol. 42, no. 9, pp. 781-785, 2010.

[84] H. Przuntek, T. Muller, and P. Riederer, "Diagnostic staging of Parkinson's disease: conceptual aspects," Journal of Neural Transmission, vol. 111, no. 2, pp. 201-216, 2004.

[85] C. Weller, N. Oxlade, S. M. Dobbs, R. J. Dobbs, A. Charlett, and I. T. Bjarnason, "Role of inflammation in gastrointestinal tract in aetiology and pathogenesis of idiopathic parkinsonism," FEMS Immunology and Medical Microbiology, vol. 44, no. 2, pp. 129-135, 2005.

[86] Y. Baba, A. Kuroiwa, R. J. Uitti, Z. K. Wszolek, and T. Yamada, "Alterations of T-lymphocyte populations in Parkinson disease," Parkinsonism and Related Disorders, vol. 11, no. 8, pp. 493-498, 2005.

[87] H. Akiyama, S. Itagaki, and P. L. McGeer, "Major histocompatibility complex antigen expression on rat microglia following epidural kainic acid lesions," Journal of Neuroscience Research, vol. 20, pp. 147-157, 1988.

[88] V. Brochard, B. Combadiere, A. Prigent et al., "Infiltration of CD4+ lymphocytes into the brain contributes to neurodegeneration in a mouse model of Parkinson disease," Journal of Clinical Investigation, vol. 119, no. 1, pp. 182-192, 2009.

[89] D. K. Stone, A. D. Reynolds, R. L. Mosley, and H. E. Gendelman, "Innate and adaptive immunity for the pathobiology of Parkinson's disease," Antioxidants and Redox Signaling, vol. 11, no. 9, pp. 2151-2166, 2009.

[90] M. G. Tansey and M. S. Goldberg, "Neuroinflammation in Parkinson's disease: its role in neuronal death and implications for therapeutic intervention," Neurobiology of Disease, vol. 37, no. 3, pp. 510-518, 2010.

[91] H. Chen, S. M. Zhang, M. A. Hernan et al., "Nonsteroidal anti-inflammatory drugs and the risk of Parkinson disease," Archives of Neurology, vol. 60, no. 8, pp. 1059-1064, 2003.

[92] H. Chen, E. Jacobs, M. A. Schwarzschild et al., "Nonsteroidal antiinflammatory drug use and the risk for Parkinson's disease," Annals of Neurology, vol. 58, no. 6, pp. 963-967, 2005.

[93] T. M. Tikka and J. E. Koistinaho, "Minocycline provides neuroprotection against $\mathrm{N}$-methyl-D-aspartate neurotoxicity by inhibiting microglia," Journal of Immunology, vol. 166, no. 12, pp. 7527-7533, 2001

[94] H. S. Kim and Y. H. Suh, "Minocycline and neurodegenerative diseases," Behavioural Brain Research, vol. 196, no. 2, pp. 168-179, 2009.
[95] M. Tomas-Camardiel, I. Rite, A. J. Herrera et al., "Minocycline reduces the lipopolysaccharide-induced inflammatory reaction, peroxynitrite-mediated nitration of proteins, disruption of the blood-brain barrier, and damage in the nigral dopaminergic system," Neurobiology of Disease, vol. 16, no. 1, pp. 190-201, 2004.

[96] L. M. Kosloski, D. M. Ha, J. A. L. Hutter et al., "Adaptive immune regulation of glial homeostasis as an immunization strategy for neurodegenerative diseases," Journal of Neurochemistry, vol. 114, no. 5, pp. 1261-1276, 2010.

[97] M. J. Kluger, "Fever: role of pyrogens and cryogens," Physiological Reviews, vol. 71, no. 1, pp. 93-127, 1991.

[98] P. M. Carvey, Q. Chang, J. W. Lipton, and Z. Ling, "Prenatal exposure to the bacteriotoxin lipopolysaccharide leads to long-term losses of dopamine neurons in offspring: a potential, new model of Parkinson's disease," Frontiers in Bioscience, vol. 8, pp. s826-s837, 2003.

[99] Z. D. Ling, D. A. Gayle, S. Y. Ma et al., "In utero bacterial endotoxin exposure causes loss of tyrosine hydroxylase neurons in the postnatal rat midbrain," Movement Disorders, vol. 17, no. 1, pp. 116-124, 2002.

[100] Z. Ling, Q. A. Chang, C. W. Tong, S. E. Leurgans, J. W. Lipton, and P. M. Carvey, "Rotenone potentiates dopamine neuron loss in animals exposed to lipopolysaccharide prenatally," Experimental Neurology, vol. 190, no. 2, pp. 373-383, 2004.

[101] H. Engler, R. Doenlen, C. Riether et al., "Time-dependent alterations of peripheral immune parameters after nigrostriatal dopamine depletion in a rat model of Parkinson's disease," Brain, Behavior, and Immunity, vol. 23, no. 4, pp. 518-526, 2009.

[102] R. F. Villarán, A. M. Espinosa-Oliva, M. Sarmiento et al., "Ulcerative colitis exacerbates lipopolysaccharide-induced damage to the nigral dopaminergic system: potential risk factor in Parkinson's disease," Journal of Neurochemistry, vol. 114, no. 6, pp. 1687-1700, 2010.

[103] D. C. Anthony, S. J. Bolton, S. Fearn, and V. H. Perry, "Age-related effects of interleukin- $1 \beta$ on polymorphonuclear neutrophil-dependent increases in blood-brain barrier permeability in rats," Brain, vol. 120, no. 3, pp. 435-444, 1997.

[104] C. C. Ferrari, A. M. Depino, F. Prada et al., "Reversible demyelination, blood-brain barrier breakdown, and pronounced neutrophil recruitment induced by chronic IL-1 expression in the brain," American Journal of Pathology, vol. 165, no. 5, pp. 1827-1837, 2004.

[105] C. C. Ferrari, M. C. Pott Godoy, R. Tarelli, M. Chertoff, A. M. Depino, and F. J. Pitossi, "Progressive neurodegeneration and motor disabilities induced by chronic expression of IL- $1 \beta$ in the substantia nigra," Neurobiology of Disease, vol. 24, no. 1, pp. 183-193, 2006.

[106] S. S. Shaftel, T. J. Carlson, J. A. Olschowka, S. Kyrkanides, S. B. Matousek, and M. K. O’Banion, “Chronic interleukin-1 $\beta$ expression in mouse brain leads to leukocyte infiltration and neutrophil-independent blood-brain barrier permeability without overt neurodegeneration," Journal of Neuroscience, vol. 27, no. 35, pp. 9301-9309, 2007.

[107] S. J. Campbell, D. C. Wilcockson, A. G. Butchart, V. H. Perry, and D. C. Anthony, "Altered chemokine expression in the spinal cord and brain contributes to differential interleukin- $1 \beta$-induced neutrophil recruitment," Journal of Neurochemistry, vol. 83, no. 2, pp. 432-441, 2002.

[108] S. Ching, L. He, W. Lai, and N. Quan, "IL-1 type I receptor plays a key role in mediating the recruitment of leukocytes into the central nervous system," Brain, Behavior, and Immunity, vol. 19, no. 2, pp. 127-137, 2005. 
[109] L. Qin, X. Wu, M. L. Block et al., "Systemic LPS causes chronic neuroinflammation and progressive neurodegeneration," GLIA, vol. 55, no. 5, pp. 453-462, 2007.

[110] M. K. McCoy, T. N. Martinez, K. A. Ruhn et al., "Blocking soluble tumor necrosis factor signaling with dominant-negative tumor necrosis factor inhibitor attenuates loss of dopaminergic neurons in models of Parkinson's disease," Journal of Neuroscience, vol. 26, no. 37, pp. 9365-9375, 2006.

[111] A. D. Reynolds, R. Banerjee, J. Liu, H. E. Gendelman, and R. L. Mosley, "Neuroprotective activities of CD4+CD25+ regulatory T cells in an animal model of Parkinson's disease," Journal of Leukocyte Biology, vol. 82, no. 5, pp. 1083-1094, 2007.

[112] A. D. Reynolds, D. K. Stone, J. A. L. Hutter, E. J. Benner, R. L. Mosley, and H. E. Gendelman, "Regulatory T cells attenuate Th17 cell-mediated nigrostriatal dopaminergic neurodegeneration in a model of Parkinson's disease," Journal of Immunology, vol. 184, no. 5, pp. 2261-2271, 2010.

[113] X. Huang, A. D. Reynolds, R. L. Mosley, and H. E. Gendelman, "CD 4+ T cells in the pathobiology of neurodegenerative disorders," Journal of Neuroimmunology, vol. 211, no. 1-2, pp. 3-15, 2009.

[114] I. Kurkowska-Jastrzebska, M. Babiuch, I. Joniec, A. Przybylkowski, A. Czlonkowski, and A. Czlonkowska, "Indomethacin protects against neurodegeneration caused by MPTP intoxication in mice," International Immunopharmacology, vol. 2, no. 8, pp. 1213-1218, 2002.

[115] P. Teismann, K. Tieu, D. K. Choi et al., "Cyclooxygenase-2 is instrumental in Parkinson's disease neurodegeneration," Proceedings of the National Academy of Sciences of the United States of America, vol. 100, no. 9, pp. 5473-5478, 2003.

[116] P. Teismann, M. Vila, D. K. Choi et al., "COX-2 and neurodegeneration in Parkinson's disease," Annals of the New York Academy of Sciences, vol. 991, pp. 272-277, 2003.

[117] R. Sanchez-Pernaute, A. Ferree, O. Cooper, M. Yu, A. L. Brownell, and O. Isacson, "Selective COX-2 inhibition prevents progressive dopamine neuron degeneration in a rat model of Parkinson's disease," Journal of Neuroinflammation, vol. 1, article 6, 2004. 


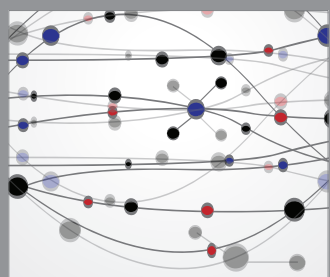

The Scientific World Journal
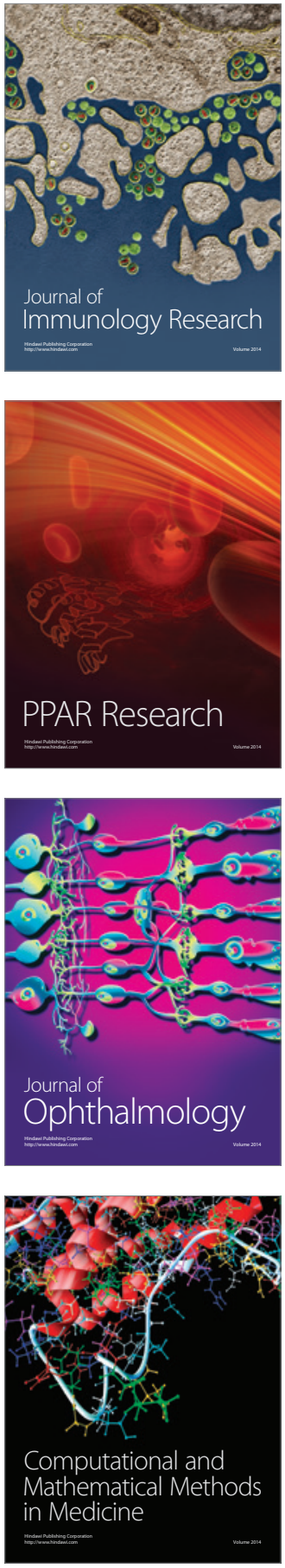

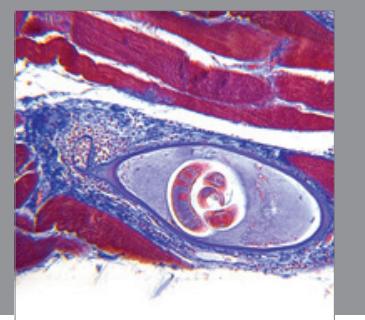

Gastroenterology

Research and Practice
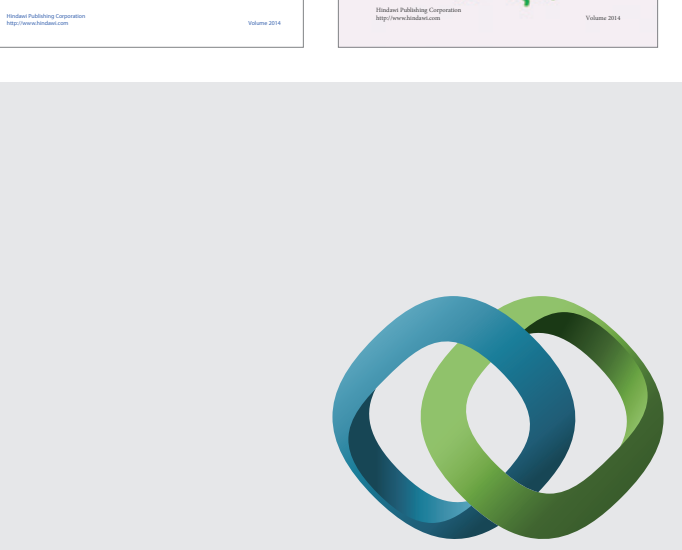

\section{Hindawi}

Submit your manuscripts at

http://www.hindawi.com
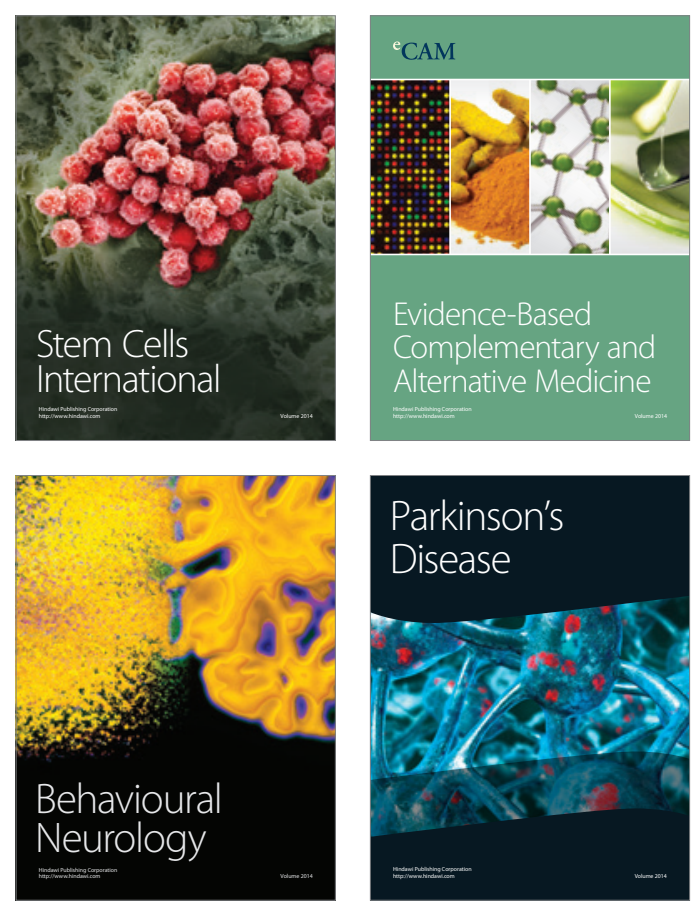

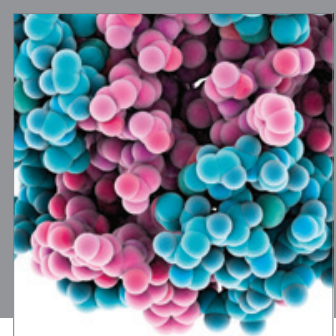

Journal of
Diabetes Research

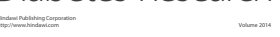

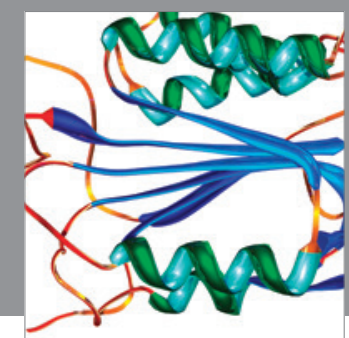

Disease Markers
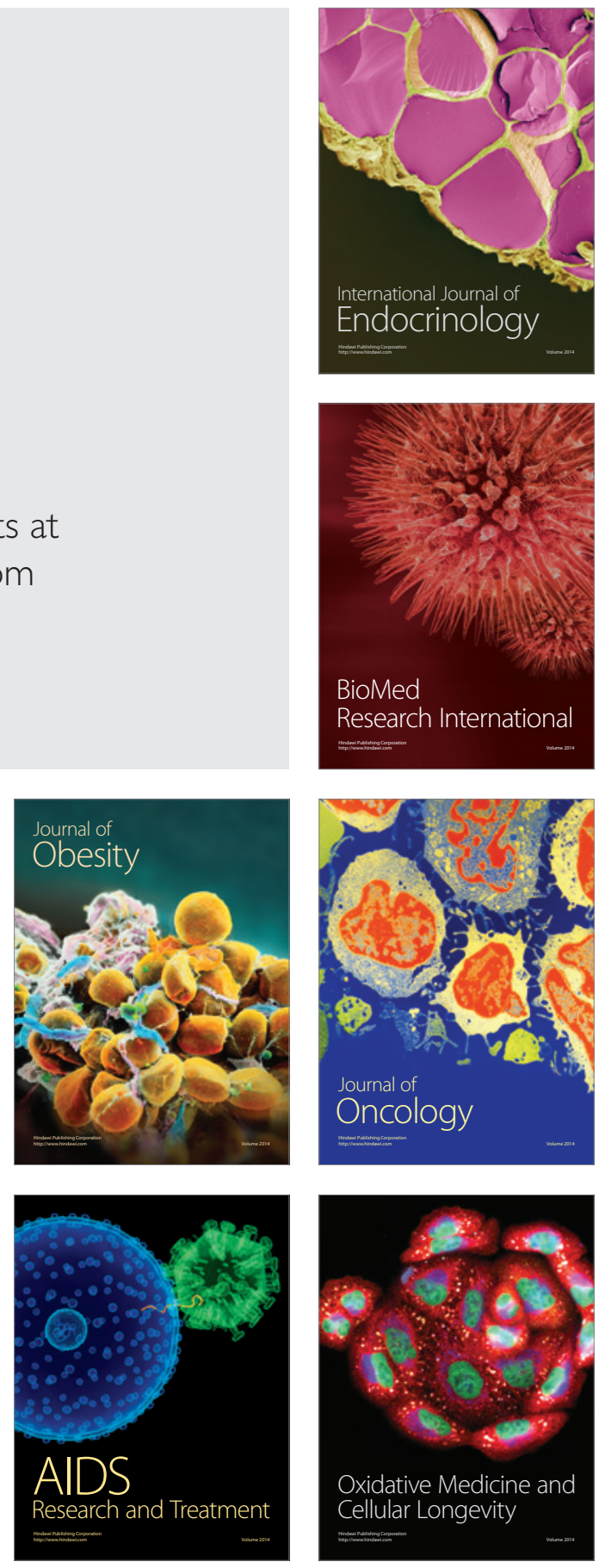